\title{
Review of: "Structural basis for the peptidoglycan editing activity of YfiH"
}

\author{
Ramaswamy Subramanian ${ }^{1}$
}

1 Purdue University

Potential competing interests: The author(s) declared that no potential competing interests exist.

The manuscript describes a very well-done piece of work where the authors show the function of YfiH. It was shown earlier that YfiH absence increases the incorporation of L-Ser into the peptidoglycan. However, how $\mathrm{YfiH}$ manages to do this function was not clear.

Using a serendipitous finding in the crystal structure of the active site C107 mutation - where they found a bound UDP-MurNAc, and the presence of water molecules, they hypothesized that the substrate is UDPMurNAc-Ser. By a set of clever biochemical experiments, they prove their point. Along the way, they also identify the critical residue for catalysis.

An interesting technique is using the catalytically active $\mathrm{YfiH}$ as an additive in the biosynthesis of UMG by MurC. They show the higher selectivity of YfiH to cleaving when glycine is incorporated.

The structures are of high resolution, and the enzyme assays and mass-spectrometry data are sound. Several structures of YfiH proteins in the PDB are not published as its natural function was not known. Here they show the proofreading function for peptidoglycan biosynthesis as the primary function of YfiH. 